\title{
WEIGHTED SOBOLEV SPACES AND PSEUDODIFFERENTIAL OPERATORS WITH SMOOTH SYMBOLS
}

\author{
BY
}

\author{
NICHOLAS MILLER
}

\begin{abstract}
Let $u^{\#}$ be the Fefferman-Stein sharp function of $u$, and for $1<r<\infty$, let $M_{r} u$ be an appropriate version of the Hardy-Littlewood maximal function of $u$. If $\boldsymbol{A}$ is a (not necessarily homogeneous) pseudodifferential operator of order 0 , then there is a constant $c>0$ such that the pointwise estimate $(A u)^{\#}(x) \leqslant c M_{r} u(x)$ holds for all $x \in R^{n}$ and all Schwartz functions $u$. This estimate implies the boundedness of 0 -order pseudodifferential operators on weighted $L^{p}$ spaces whenever the weight function belongs to Muckenhoupt's class $A_{p}$. Having established this, we construct weighted Sobolev spaces of fractional order in $R^{n}$ and on a compact manifold, prove a version of Sobolev's theorem, and exhibit coercive weighted estimates for elliptic pseudodifferential operators.
\end{abstract}

1. Introduction. In this paper, we prove boundedness results for pseudodifferential operators on weighted $L^{p}$ spaces. The methods are different from those which have appeared in the literature and depend upon a pointwise estimate proved in Theorem 2.8. Since this estimate does not rely on properties of weight functions, it is of independent interest and may be of further use in discovering how pseudodifferential operators preserve various classes of functions and their differentiability properties.

If $1<p<\infty$, a nonnegative function $w$ belongs to $A_{p}\left(R^{n}\right)$ if:

(i) $w \in L_{\mathrm{loc}}^{1}\left(R^{n}\right)$;

(ii)

$$
\sup _{Q}\left(\frac{1}{|Q|} \int_{Q} w d x\right)\left(\frac{1}{|Q|} \int_{Q} w^{-1 /(p-1)} d x\right)^{p-1}<\infty,
$$

where the supremum is taken over all cubes $Q$ in $R^{n}$.

Coifman, Fefferman, Hunt, Muckenhoupt, and Wheeden have shown [2], [4], [6] that a weight function $w$ satisfies the $A_{p}$ condition if and only if the HardyLittlewood maximal operator or classical singular integral operators are bounded on $L^{p}\left(R^{n}, w d x\right)$. Our boundedness results for pseudodifferential operators will also apply to spaces with $A_{p}$ weight functions.

We shall say that the function $a(x, \xi) \in C^{\infty}\left(R^{n} \times R^{n}\right)$ is a symbol of order $m$ if it satisfies the estimates

$$
\left|\left(\frac{\partial}{\partial x}\right)^{\alpha}\left(\frac{\partial}{\partial \xi}\right)^{\beta} a(x, \xi)\right| \leqslant C_{\alpha \beta}(1+|\xi|)^{m-|\beta|}
$$

Received February 26, 1980 and, in revised form, January 16, 1981. Presented at the January, 1980 meeting of the AMS in San Antonio, Texas.

1980 Mathematics Subject Classification. Primary 35S05, 46E35, 47G05; Secondary 58G15.

Key words and phrases. $A_{p}$ weight, maximal function, pseudodifferential operator, Sobolev space. 
for all multi-indices $\alpha$ and $\beta$. A symbol of order $-\infty$ is one which satisfies the above estimates for each real number $m$. If $a(x, \xi)$ is a symbol of order $m$, then it defines a pseudodifferential operator $A$, of order $m$, by the formula

$$
A u(x)=\int_{R^{n}} a(x, \xi) \hat{u}(\xi) e^{2 \pi i x \cdot \xi} d \xi .
$$

To begin with, $A$ is defined only on the space of Schwartz functions, where the Fourier transform $\hat{u}$ of the function $u$ is given by

$$
\hat{u}(\xi)=\int_{R^{n}} u(x) e^{-2 \pi i x \cdot \xi} d x .
$$

That $A$ can be extended to a larger class of functions is the main result of this paper. Theorems 2.2 and 2.12 contain this result, which we summarize as:

Suppose $1<p<\infty$. Every pseudodifferential operator of order 0 has a bounded extension to $L^{p}\left(R^{n}, w d x\right)$ if and only if $w \in A_{p}\left(R^{n}\right)$.

The necessity of the $A_{p}$ condition is proved using a modification of an argument by Coifman and Fefferman. The sufficiency is proved by controlling the pseudodifferential operator with various versions of the Hardy-Littlewood maximal operator, which appeared in [3]. With this goal in mind, we make the following definitions:

$$
M f(x)=\text { the Hardy-Littlewood maximal function of } f=\sup _{Q} \frac{1}{|Q|} \int_{Q}|f(y)| d y,
$$

the supremum being taken over all cubes $Q$ containing $x$;

$$
M_{r} f(x)=\sup _{Q}\left(\frac{1}{|Q|} \int_{Q}|f(y)|^{r} d y\right)^{1 / r},
$$

the supremum being taken over all cubes $Q$ containing $x$;

(c)

$$
f^{*}(x)=\text { the dyadic maximal function of } f=\sup _{Q} \frac{1}{|Q|} \int_{Q}|f(y)| d y,
$$

the supremum being taken over all dyadic cubes $Q$, with sides parallel to the axes, containing $x$;

(d)

$$
f^{\#}(x)=\sup _{Q} \frac{1}{|Q|} \int_{Q}\left|f(y)-f_{Q}\right| d y,
$$

where the supremum is taken over all cubes $Q$ containing $x$, and $f_{Q}$ is the average value of $f$ on the cube $Q$.

Note that $f^{*}$ enjoys many of the properties of the more usual maximal function $M f$; in particular, $|f(x)| \leqslant f^{*}(x)$ a.e., and the operator $f \rightarrow f^{*}$ is bounded on $L^{p}\left(R^{n}, w d x\right)$ whenever $1<p<\infty$ and $w \in A_{p}\left(R^{n}\right)$ [2].

In addition to all the foregoing maximal function machinery, the proof of the main result requires the following pointwise estimate, which is the one mentioned at the beginning of this introduction. 
THEOREM 2.8. Suppose $1<r<\infty$, and let $A$ be a pseudodifferential operator of order 0 . Then there is a constant $c>0$ such that the pointwise estimate $(A u)^{\#}(x)<$ $c M_{r} u(x)$ holds for all $x \in R^{n}$ and all Schwartz functions $u$.

Armed with these two theorems, we then define weighted Sobolev spaces in $R^{n}$ and prove the usual a priori estimates of elliptic differential operators. We also formulate the $A_{p}$ condition for a compact manifold without boundary and show that the condition is invariant under coordinate changes. In the setting of a manifold, further results are the construction of weighted Sobolev spaces of fractional order, a version of Sobolev's theorem, and coercive estimates for elliptic pseudodifferential operators.

REMARK. Note that the theorem above has been proved for classical singular integral operators by Cordoba and Fefferman $[A$ weighted norm inequality for singular integrals, Studia Math. 57 (1976), 97-101]. Our theorem shows that the method works for "variable coefficient" operators defined by nonhomogeneous kernels and that these operators can be used to give painless constructions of weighted Sobolev spaces.

2. $L^{p}$ estimates in $R^{n}$. Until further notice, $\|\cdot\|_{p}$ will denote the norm in the space $L^{p}\left(R^{n}, w d x\right) ; w$ will always be a weight function of class $A_{p}\left(R^{n}\right)$. We shall prove estimates of the form $\|A u\|_{p} \leqslant c\|u\|_{p}$ for $u$ a Schwartz function and $A$ a pseudodifferential operator of order 0 . The next lemma shows that once this is done, $A$ can be defined as a bounded operator on $L^{p}\left(R^{n}, w d x\right)$.

LEMMA 2.1. $\delta$, the set of all Schwartz functions, is dense in $L^{p}\left(R^{n}, w d x\right)$, $1<p<\infty$.

Proof. We first show that smooth functions with compact support are dense in $L^{p}$. Given $f$ in $L^{p}$ and $\varepsilon>0$, choose a continuous function $g$ with compact support such that $\|f-g\|_{p}<\varepsilon / 2[8$, Theorem 3.14].

Now let $\phi$ be a positive-valued $C^{\infty}$ function supported in the unit ball of $R^{n}$ with total integral 1. Define

$$
\phi_{t}(x)=t^{-n} \phi(x / t) \text { for } t>0 .
$$

It is standard knowledge that

(a) $\phi_{t} * g \in C_{0}^{\infty}\left(R^{n}\right)$ for all $t>0$, and

(b) $\phi_{t} * g \rightarrow g$, as $t \rightarrow 0$, uniformly on compact subsets of $R^{n}$.

If $K$ is a large ball containing the support of $g$ in its interior, pick $t$ small enough that

$$
\left\|g-\phi_{t} * g\right\|_{\infty}<\frac{\varepsilon}{2}\left(\int_{K} w d x\right)^{-1 / p}
$$

Then

$$
\begin{aligned}
\left\|f-\phi_{t} * g\right\|_{p} & \leqslant\|f-g\|_{p}+\left\|g-\phi_{t} * g\right\|_{p} \\
& <\varepsilon / 2+\varepsilon / 2=\varepsilon ;
\end{aligned}
$$

this shows that $C_{0}^{\infty}\left(R^{n}\right)$ is dense in $L^{p}\left(R^{n}, w d x\right)$. 
It remains only to show that $\delta \subset L^{p}\left(R^{n}, w d x\right)$. If $w \in A_{p}\left(R^{n}\right)$, then Lemma 1 of [4] implies that $\int_{R^{n}} w(x) /(1+|x|)^{k} d x<\infty$ for large enough $k$. But if $u \in \mathcal{S}$, then $|u(x)| \leqslant C_{k} /(1+|x|)^{k / p}$, which proves the assertion in the first sentence of this paragraph.

The $A_{p}$ condition is a necessary one for continuity of even the best-behaved pseudodifferential operators; the proof of this fact is adapted from [2]. From now on, if $p$ is a real number between 1 and $\infty, p^{\prime}$ will denote its conjugate, the number such that $1 / p+1 / p^{\prime}=1$.

THEOREM 2.2. Suppose $w$ is a nonnegative locally integrable function whose zero-set has Lebesgue measure 0 . If every pseudodifferential operator of order $-\infty$ is continuous on $L^{p}\left(R^{n}, w d x\right)$, then $w \in A_{p}\left(R^{n}\right), 1<p<\infty$.

Proof. We first establish that $w^{-1 /(p-1)} \in L^{1}(Q, d x)$ for any cube $Q$ in $R^{n}$. Suppose $Q$ is a cube such that $w^{-1 /(p-1)} \notin L^{1}(Q, d x)$; then $w^{-1 / p} \notin L^{p^{\prime}}(Q, d x)$, and there is a function $\phi \in L^{p}(Q, d x)$ such that $\int_{Q} \phi w^{-1 / p} d x=\infty$. Let $\psi=$ $\phi w^{-1 / p}$, and let $\tau \in C_{0}^{\infty}\left(R^{n}\right)$ have the value 1 in the set $Q-Q=\{x-y$ : $x, y \in Q\}$. The operator $T u=\tau * u$ is a pseudodifferential operator of order $-\infty$ (its symbol, $\hat{\tau}$, is rapidly decreasing), and by hypothesis it is continuous on $L^{p}\left(R^{n}, w d x\right)$. Now $\psi \in L^{p}\left(R^{n}, w d x\right)$, since $\phi$ is supported in $Q$, but $T \psi(x)=\infty$ for almost all $x \in Q$. This is impossible since $w$ has a zero-set of Lebesgue measure 0 . Hence $w^{-1 /(p-1)} \in L^{1}(Q, d x)$.

Having disposed of this preliminary step, we now show the necessity of the $A_{p}$ condition. Fix a cube $Q$ of side length $d$, and let $Q^{\prime}$ be an adjoining cube of the same size. If $x \in Q$ and $y \in Q^{\prime}$, then $|x-y| \leqslant 2 \sqrt{n} d$. Suppose $g \in C_{0}^{\infty}\left(R^{n}\right)$, $g \geqslant 0$, and $g(x)=1 / d^{n}$ if $|x| \leqslant 3 \sqrt{n} d$. Again, $f \rightarrow f * g$ is a pseudodifferential operator of order $-\infty$ since $\hat{g} \in \mathcal{\delta}$.

If $f \geqslant 0$ is supported in $Q$, then

$$
f * g(x)=\int_{Q} f(y) g(x-y) d y \geqslant\left(\frac{1}{|Q|} \int_{Q} f(y) d y\right) \chi_{Q^{\prime}}(x) .
$$

Hence,

$$
\left(\int_{Q^{\prime}} w d x\right)\left(\frac{1}{|Q|} \int_{Q} f(y) d y\right)^{p} \leqslant \int_{Q^{\prime}}|f * g|^{p} w d x \leqslant C \int_{Q} f^{p} w d x,
$$

the last inequality being a consequence of the assumption on $w$.

Now let $f \equiv 1$ on $Q$ to get $\int_{Q^{\prime}} w d x \leqslant C \int_{Q} w d x$; interchanging $Q$ and $Q^{\prime}$, we get $\int_{Q} w d x \leqslant C \int_{Q^{\prime}} w d x$.

Since $w^{-1 /(p-1)} \in L^{p}(Q, w d x)$ by the first part of the proof, we can now let $f=w^{-1 /(p-1)} \lambda_{Q}$

$$
\begin{aligned}
\left(\frac{1}{|Q|} \int_{Q} w d x\right)\left(\frac{1}{|Q|} \int_{Q} w^{-1 /(p-1)} d x\right)^{p} & \leqslant C\left(\frac{1}{\left|Q^{\prime}\right|} \int_{Q^{\prime}} w d x\right)\left(\frac{1}{|Q|} \int_{Q} w^{-1 /(p-1)} d x\right)^{p} \\
& \leqslant \frac{C}{|Q|} \int_{Q} w^{-1 /(p-1)} d x
\end{aligned}
$$


by (2.3). Hence,

$$
\left(\frac{1}{|Q|} \int_{Q} w d x\right)\left(\frac{1}{|Q|} \int_{Q} w^{-1 /(p-1)} d x\right) \leqslant C .
$$

Having established the necessity of the $A_{p}$ condition for boundedness of pseudodifferential operators, we can now turn to the sufficiency of the condition.

LEMMA 2.4. Suppose $1<p<\infty$. Let $\psi$ be a radial, decreasing, positive function with total integral 1. Set $\psi_{t}(x)=t^{-n} \psi(x / t)$. Then:

(i) $\sup _{t>0}\left|f * \psi_{t}(x)\right| \leqslant M f(x)$ for $f \in L^{p}\left(R^{n}, w d x\right)$;

(ii) if $\psi$ has compact support, then $f * \psi_{t}(x) \rightarrow f(x)$, as $t \rightarrow 0$, almost everywhere for $f \in L^{p}\left(R^{n}, w d x\right)$;

(iii) if $\psi$ has compact support, then $\left\|f * \psi_{t}-f\right\|_{p} \rightarrow 0$ as $t \rightarrow 0$, for all $f \in$ $L^{p}\left(R^{n}, w d x\right)$.

Proof. A proof of (i) can be found on page 63 of [9].

The proof of (ii) is adapted from page 64 of [9]. Let $B$ be any ball in $R^{n}$, let $B^{\prime}$ be any other ball containing $B$ in its interior, and let $\delta$ be the distance from $B$ to the complement of $B^{\prime}$. We shall show that if $f \in L^{p}\left(R^{n}, w d x\right)$ then $f * \psi_{t}(x) \rightarrow f(x)$ as $t \rightarrow 0$ for almost every $x \in B$. Then, by expanding $B$, we establish (ii) for almost every $x \in R^{n}$.

Set $f_{1}(x)$ equal to $f(x)$ if $x \in B^{\prime}$, and equal to 0 outside $B^{\prime}$. Let $f_{2}=f-f_{1}$. Now, $f_{1} \in L^{1}\left(R^{n}, d x\right)$ since

$$
\int_{B^{\prime}}\left|f_{1}\right| d x \leqslant\left(\int_{B^{\prime}}\left|f_{1}\right|^{p} w d x\right)^{1 / p}\left(\int_{B^{\prime}} w^{-1 /(p-1)} d x\right)^{1 / p^{\prime}}<\infty .
$$

The last integral is finite since $w^{-1 /(p-1)}$ is locally integrable.

Hence $f_{1} * \psi_{t}(x) \rightarrow f_{1}(x)=f(x)$ as $t \rightarrow 0$ for almost every $x \in B$ by part (b) of Theorem 2 on page 62 of [9].

To deal with $f_{2}$, we note that if $x \in B$, then

$$
\begin{aligned}
\left|f_{2} * \psi_{t}(x)\right| \leqslant & \int \psi_{t}(x-y)\left|f_{2}(y)\right| d y \\
\leqslant & \left(\int\left|f_{2}(y)\right|^{p} w(y) d y\right)^{1 / p} \\
& \cdot\left(\int_{|x-y|>\delta} \psi_{t}(x-y)^{\left.p^{\prime} w^{-1 /(p-1)}(y) d y\right)^{1 / p^{\prime}}}\right. \\
= & 0
\end{aligned}
$$

for sufficiently small $t$, since $\psi$ has compact support. This completes the proof of (ii).

Part (iii) is now easy, since $\left|\psi_{t} * f-f\right| \leqslant M f+|f|$ by part (i). Since $M f \in$ $L^{p}\left(R^{n}, w d x\right)$ [2], Lebesgue's dominated convergence theorem and (ii) at once yield a proof of (iii).

Now we can use the Hardy-Littlewood maximal operator to dominate any pseudodifferential operator of order $-\infty$. 
THEOREM 2.5. Suppose $A$ is a pseudodifferential operator of order $-\infty$, and suppose $1<r<\infty$. Then there exists a constant $c>0$ such that for all $x^{0} \in R^{n}$ and all $u \in \delta$,

$$
(A u)^{\#}\left(x^{0}\right) \leqslant c M_{r} u\left(x^{0}\right) .
$$

Proof. If $a(x, \xi)$ is the symbol of $A$, then for any real number $m$, and any multi-indices $\alpha$ and $\beta$,

$$
\left|\left(\frac{\partial}{\partial x}\right)^{\alpha}\left(\frac{\partial}{\partial \xi}\right)^{\beta} a(x, \xi)\right| \leqslant C_{\alpha \beta m}(1+|\xi|)^{m} .
$$

We can therefore write the operator as follows: for any Schwartz function $u$,

$$
A u(x)=\int \hat{u}(\xi) a(x, \xi) e^{2 \pi i x \cdot \xi} d \xi=\int u(y) K(x, x-y) d y,
$$

where

$$
K(x, y)=\int a(x, \xi) e^{2 \pi i y \cdot \xi} d \xi .
$$

Note that for fixed $x, K(x, y)$, as a function of $y$, lies in $\delta$. In fact,

$$
\begin{aligned}
\left|y^{\alpha}\left(\frac{\partial}{\partial y}\right)^{\beta} K(x, y)\right| & =C_{\alpha \beta}\left|\int a(x, \xi) \xi^{\beta}\left(\frac{\partial}{\partial \xi}\right)^{\alpha} e^{2 \pi y \cdot \xi} d \xi\right| \\
& \leqslant C_{\alpha \beta} \int\left|\left(\frac{\partial}{\partial \xi}\right)^{\alpha}\left[a(x, \xi) \xi^{\beta}\right]\right| d \xi \\
& \leqslant C_{\alpha \beta}, \quad \text { (integration by parts) }
\end{aligned}
$$

with $C_{\alpha \beta}$ independent of $x$ and $y$. The rapid decrease in $\xi$ of $a(x, \xi)$ justifies the differentiation under the integral sign and the integrations by parts in the calculation above. Hence,

$$
\sup _{x, y}\left|y^{\alpha}\left(\frac{\partial}{\partial y}\right)^{\beta} K(x, y)\right| \leqslant C_{\alpha \beta} .
$$

Now choose an integer $k>n$. By the previous discussion, there is a constant $C_{k}>0$ such that

$$
|K(x, y)| \leqslant C_{k} /(1+|y|)^{k} \text { for all } x .
$$

Then

$$
\begin{aligned}
|A u(x)| & \leqslant \int|u(y)||K(x, x-y)| d y \\
& \leqslant C_{k} \int \frac{|u(y)|}{(1+|x-y|)^{k}} d y \leqslant C_{k} M u(x),
\end{aligned}
$$

by an application of Lemma 2.4 .

Suppose that $x^{0}$ is any point in $R^{n}$, that $Q$ is a cube containing $x^{0}$ in its interior, and that $Q$ has diameter $d$ and center $x^{\prime}$. Let $\tau \in C_{0}^{\infty}\left(R^{n}\right)$ satisfy $0<\tau(x)<1$, be 
1 when $\left|x-x^{\prime}\right| \leqslant 2 d$, and vanish when $\left|x-x^{\prime}\right| \geqslant 3 d$. We have

$$
\begin{aligned}
\frac{1}{|Q|} \int_{Q}\left|A u(x)-(A u)_{Q}\right| d x & \leqslant \frac{2}{|Q|} \int_{Q}|A u(x)| d x \\
& \leqslant \frac{2}{|Q|} \int_{Q}|A(\tau u)| d x+\frac{2}{|Q|} \int_{Q}|A((1-\tau) u)| d x .
\end{aligned}
$$

Let $Q^{\prime}$ be the cube centered at $x^{\prime}$, with sides parallel to those of $Q$, and with diameter $4 d$. Since the Hardy-Littlewood maximal operator is bounded on $L^{r}\left(R^{n}, d x\right)$ for $1<r<\infty$, we can control the first term in the inequality above as follows:

$$
\begin{aligned}
\frac{2}{|Q|} \int_{Q}|A(\tau u)| d x & \leqslant 2\left(\frac{1}{|Q|} \int_{Q}|A(\tau u)|^{r} d x\right)^{1 / r} \\
& \leqslant 2\left(\frac{1}{|Q|} \int_{Q}|M(\tau u)|^{r} d x\right)^{1 / r} \leqslant C_{r}\left(\frac{1}{|Q|} \int_{R^{n}}|\tau u|^{r} d x\right)^{1 / r} \\
& \leqslant C_{r}\left(\frac{1}{\left|Q^{\prime}\right|} \int_{Q^{\prime}}|u|^{r} d x\right)^{1 / r} \leqslant C_{r} M_{r} u\left(x^{0}\right) .
\end{aligned}
$$

To dominate the other term, we first note that there is a constant $c>0$ such that $\left|x^{0}-y\right| \leqslant c|x-y|$ for all $x \in Q$ and $y$ such that $\left|x^{\prime}-y\right| \geqslant 2 d$. The constant $c$ is independent of $x, y$, and the cube $Q$. So,

$$
\begin{aligned}
\frac{2}{|Q|} \int_{Q}|A((1-\tau) u)| d x & =\frac{2}{|Q|} \int_{Q}\left|\int(1-\tau(y)) u(y) K(x, x-y) d y\right| d x \\
& \leqslant \int_{\left|y-x^{\prime}\right|>2 d}|u(y)| \frac{1}{|Q|} \int_{Q} \frac{C_{k}}{(1+|x-y|)^{k}} d x d y \\
& \leqslant C_{k} \int_{R^{n}} \frac{|u(y)|}{\left(1+\left|x^{0}-y\right|\right)^{k}} d y \\
& \leqslant c M u\left(x^{0}\right) \quad(\text { by Lemma 2.4) } \\
& \leqslant c M_{r} u\left(x^{0}\right) .
\end{aligned}
$$

We have showed that

$$
\frac{1}{|Q|} \int_{Q}\left|A u(x)-(A u)_{Q}\right| d x \leqslant c M_{r} u\left(x^{0}\right) .
$$

Taking the supremum of the left side over all cubes $Q$ containing $x^{0}$, we find that $(A u)^{\#}\left(x^{0}\right) \leqslant c M_{r} u\left(x^{0}\right)$.

COROllary 2.6. A pseudodifferential operator $A$, of order $-\infty$, has a bounded extension to $L^{p}\left(R^{n}, w d x\right)$ whenever $w \in A_{p}\left(R^{n}\right)$ and $1<p<\infty$.

Proof. In the course of proving the last theorem, we showed that $|A u(x)|<$ $c M u(x)$ for all $x \in R^{n}$ and $u \in \mathcal{S}$. The constant $c$ is independent of $x$ and $u$. Since $\delta$ is dense in $L^{p}\left(R^{n}, w d x\right)$ (Lemma 2.1), and the maximal operator is bounded on $L^{p}\left(R^{n}, w d x\right)[2$, Theorem I], the conclusion of the corollary follows immediately. 
Dealing with operators of order 0 will require a much more delicate touch than in the previous theorem; however, the variants of the maximal function defined in the Introduction together with ever-reliable integration by parts will save the day.

We shall use $f^{\#}$ to control $f^{*}$; the next lemma makes this possible.

LEMMA 2.7. There is a constant $c>0$ such that $\left\|f^{*}\right\|_{p} \leqslant c\left\|f^{\#}\right\|_{p}$ for all $f \in$ $L^{p}\left(R^{n}, w d x\right) \cap L^{1}\left(R^{n}, d x\right)$.

Proof. Fefferman and Stein prove this as Theorem 5 of [3] for the case $w(x) \equiv 1$. Their proof relies on the fact that $w \in A_{\infty}$; since $A_{p} \subset A_{\infty}$ [2, Lemma 3], we can use their proof in our own situation.

We are ready to prove the pointwise result mentioned in the Introduction.

TheOREM 2.8. Suppose $1<r<\infty$, and let $A$ be a pseudodifferential operator of order 0 . Then there is a constant $c>0$ such that the pointwise estimate $(A u)^{\#}\left(x^{0}\right)<$ $c M_{r} u\left(x^{0}\right)$ holds for all $x^{0} \in R^{n}$ and all $u \in \mathcal{S}$.

Proof. Given $x^{0} \in R^{n}$, we let $Q$ be a cube containing $x^{0}$, with center $x^{\prime}$ and diameter $d$. As in Theorem 2.5, we also let $\tau \in C_{0}^{\infty}\left(R^{n}\right)$ satisfy $0 \leqslant \tau(x)<1$, be 1 when $\left|x-x^{\prime}\right| \leqslant 2 d$, and vanish when $\left|x-x^{\prime}\right| \geqslant 3 d$. Then for $u \in \mathcal{S}$,

$$
\begin{aligned}
\frac{1}{|Q|} \int_{Q}\left|A u(x)-(A u)_{Q}\right| d x \leqslant & \frac{2}{|Q|} \int_{Q}|A(\tau u)| d x \\
& +\frac{1}{|Q|} \int_{Q}\left|A((1-\tau) u)(x)-(A((1-\tau) u))_{Q}\right| d x .
\end{aligned}
$$

Letting $Q^{\prime}$ be as in Theorem 2.5, we can dominate the first term in the inequality above by recalling that pseudodifferential operators of order 0 are bounded on $L^{r}\left(R^{n}, d x\right)$ when $1<r<\infty[5]:$

$$
\begin{aligned}
\frac{2}{|Q|} \int_{Q}|A(\tau u)| d x & \leqslant 2\left(\frac{1}{|Q|} \int_{Q}|A(\tau u)|^{r} d x\right)^{1 / r} \leqslant c\left(\frac{1}{|Q|} \int_{R^{n}}|\tau u|^{r} d x\right)^{1 / r} \\
& \leqslant c\left(\frac{1}{\left|Q^{\prime}\right|} \int_{Q^{\prime}}|u|^{r} d x\right)^{1 / r} \leqslant c M_{r} u\left(x^{0}\right) .
\end{aligned}
$$

To deal with the second term, we simplify notation, writing $u$ for $(1-\tau) u$, and we assume that $u$ has support in the set $\left\{x:\left|x-x^{\prime}\right| \geqslant 2 d\right\}$. We must estimate the quantity $(1 /|Q|) \int_{Q}\left|A u(x)-(A u)_{Q}\right| d x$.

For now, we shall also assume that $a(x, \xi)$, the symbol of $A$, has compact $\xi$-support. The various constants that occur in the following argument will not depend on the support of $a$; at the end we show how to dispense with the assumption on the support of $a$.

We begin by decomposing the operator $A$ into a sum of simpler operators. Standard techniques allow us to construct a nonnegative, radial, $C^{\infty}$ function $\phi$, whose support is contained in the set $\left\{\xi: \frac{1}{2} \leqslant \xi \leqslant 2\right\}$, and which satisfies

$$
\sum_{j=0}^{\infty} \phi\left(2^{-j} \xi\right)= \begin{cases}1 & \text { if }|\xi|>1, \\ 0 & \text { if }|\xi|<\frac{1}{2} .\end{cases}
$$


Now we can write

$$
\begin{aligned}
A u(x)= & \int \hat{u}(\xi) a(x, \xi) e^{2 \pi i x \cdot \xi} d \xi \\
= & \int \hat{u}(\xi) a(x, \xi)\left(1-\sum_{j=0}^{\infty} \phi\left(2^{-j} \xi\right)\right) e^{2 \pi i x \cdot \xi} d \xi \\
& +\sum_{j=0}^{\infty} \int u(y) \int a(x, \xi) \phi\left(2^{-j} \xi\right) e^{2 \pi i(x-y) \cdot \xi} d \xi d y \\
= & B u(x)+\sum_{j=0}^{\infty} A_{j} u(x) .
\end{aligned}
$$

$B$ is a pseudodifferential operator whose symbol is $a(x, \xi)\left(1-\sum_{j=0}^{\infty} \phi\left(2^{-j} \xi\right)\right)$; the $\xi$-support of this symbol is always contained in the set $\{\xi:|\xi| \leqslant 1\}$. Hence $B$ has order $-\infty$, and $(B u)^{\#}\left(x^{0}\right) \leqslant c M_{r} u\left(x^{0}\right)$ by Theorem 2.5 . Since

$$
\begin{aligned}
(A u)^{\#}\left(x^{0}\right) & \leqslant(B u)^{\#}\left(x^{0}\right)+\left(\sum_{j=0}^{\infty} A_{j} u\right)^{\#}\left(x^{0}\right) \\
& \leqslant c M_{r} u\left(x^{0}\right)+\left(\sum_{j=0}^{\infty} A_{j} u\right)^{\#}\left(x^{0}\right),
\end{aligned}
$$

the next task is to examine the operators $A_{j}$.

$$
A_{j} u(x)=\int u(y) \int a(x, \xi) \phi\left(2^{-j} \xi\right) e^{2 \pi i(x-y) \cdot \xi} d \xi d y .
$$

The following lemma allows us to control the inner integral.

LEMMA 2.9. Let $q(x, \xi)$ be a symbol of order $m$, and suppose $\phi \in C_{0}^{\infty}\left(R^{n}\right)$ has support in $\left\{\xi: \frac{1}{2} \leqslant|\xi| \leqslant 2\right\}$. If $t \geqslant 0$, then there is a constant $c_{t}>0$ such that the inequality

$$
|y|^{t}\left|\int q(x, \xi) \phi\left(2^{-j} \xi\right) e^{2 \pi i y \cdot \xi} d \xi\right| \leqslant c_{t} 2^{j(n+m-t)}
$$

holds for all $x$ and $y$ in $R^{n}$ and every integer $j \geqslant 0$.

Proof. Suppose first that $t$ is a nonnegative integer. Letting

$$
|y|_{\infty}=\max \left\{\left|y_{i}\right|: 1 \leqslant i \leqslant n\right\},
$$

we have

$$
\begin{aligned}
&|y|_{\infty}^{t}\left|\int q(x, \xi) \phi\left(2^{-j} \xi\right) e^{2 \pi i y \cdot \xi} d \xi\right|=c_{t}\left|\int q(x, \xi) \phi\left(2^{-j} \xi\right)\left(\frac{\partial}{\partial \xi_{i}}\right)^{t} e^{2 \pi i y \cdot \xi} d \xi\right| \\
&\left.\quad \text { (where }\left|y_{i}\right|=\left|y_{\infty}\right|\right)
\end{aligned}
$$

(integration by parts). 
Now

$$
\begin{aligned}
\left|\left(\frac{\partial}{\partial \xi_{i}}\right)^{t}\left[q(x, \xi) \phi\left(2^{-j} \xi\right)\right]\right| & \leqslant \sum_{r+s=t} c_{r s}\left|\left(\frac{\partial}{\partial \xi_{i}}\right)^{r} q(x, \xi) 2^{-j s}\left(\left(\frac{\partial}{\partial \xi_{i}}\right)^{s} \phi\right)\left(2^{-j} \xi\right)\right| \\
& \leqslant \sum_{r+s=t} c_{r s}\left(1+2^{j}\right)^{m-r} 2^{-j s}
\end{aligned}
$$

(since the support of the expression above lies in $\left\{\xi: 2^{j-1}<|\xi|<2^{j+1}\right\}$ )

$$
\leqslant c_{t} 2^{j m-j t} \text {. }
$$

Substituting this estimate into (2.10) and integrating over the region $\left\{\xi: 2^{j-1} \leqslant\right.$ $\left.|\xi| \leqslant 2^{j+1}\right\}$ yields the desired inequality.

If $t>0$ is not an integer, say $i<t<i+1$, with $i$ an integer, then we can interpolate between the inequalities for $i$ and $i+1$.

Returning to the proof of the theorem, we now estimate $\left(\sum_{j=0}^{\infty} A_{j} u\right)^{\#}\left(x^{0}\right)$.

$$
\begin{aligned}
& \frac{1}{|Q|} \int_{Q}\left|A_{j} u(x)-\left(A_{j} u\right)_{Q}\right| d x \\
& =\frac{1}{|Q|} \int_{Q}\left|\frac{1}{|Q|} \int_{Q} A_{j} u(x)-A_{j} u(z) d z\right| d x \\
& =\frac{1}{|Q|} \int_{Q} \mid \frac{1}{|Q|} \int_{Q} \int_{R^{n}} u(y) \int_{R^{n}} \phi\left(2^{-j} \xi\right) \\
& \cdot\left[a(x, \xi) e^{2 \pi i(x-y) \cdot \xi}-a(z, \xi) e^{2 \pi i(z-y) \cdot \xi}\right] d \xi d y d z \mid d x .
\end{aligned}
$$

To estimate this last quantity, we consider two cases:

Case $1.2^{j} d \geqslant 1$. Then (2.11) is dominated by

$2 \sum_{k=1}^{\infty} \frac{1}{|Q|} \int_{Q} \int_{2^{k} d<\left|y-x^{\prime}\right|<2^{k+1} d}|u(y)|\left|\int_{R^{n}} \phi\left(2^{-j} \xi\right) a(x, \xi) e^{2 \pi i(x-y) \cdot \xi} d \xi\right| d y d x$

$$
\begin{aligned}
< & C \sum_{k=1}^{\infty} \int_{Q} \frac{2^{n k}}{\left|Q_{k}\right|} \int_{2^{k} d<\left|y-x^{\prime}\right|<2^{k+1} d} \frac{|u(y)|}{|x-y|^{n+1}} \cdot|x-y|^{n+1} \\
& \cdot\left|\int_{R^{n}} \phi\left(2^{-j} \xi\right) a(x, \xi) e^{2 \pi i(x-y) \cdot \xi} d \xi\right| d y d x
\end{aligned}
$$

( $Q_{k}$ is the cube with center $x^{\prime}$, sides parallel to those of $Q$, and radius $2^{k+1} d$ )

$$
\leqslant C \sum_{k=1}^{\infty} d^{n} 2^{n k}\left(2^{k} d\right)^{-n-1} 2^{-j} \cdot \frac{1}{\left|Q_{k}\right|} \int_{Q_{k}}|u(y)| d y
$$

(by Lemma 2.9 with $t=n+1$ and $m=0$ )

$$
\begin{aligned}
& \leqslant C M u\left(x^{0}\right) \sum_{k=1}^{\infty} d^{-1} 2^{-k} 2^{-j} \\
& \leqslant C d^{-1} 2^{-j} M u\left(x^{0}\right) .
\end{aligned}
$$


Case $2.2^{j} d<1$. We write

$$
\begin{aligned}
a(x, \xi) e^{2 \pi i(x-y) \cdot \xi}-a(z, \xi) e^{2 \pi i(z-y) \cdot \xi}= & \sum_{l=1}^{n}\left(x_{l}-z_{l}\right) \int_{0}^{1} \frac{\partial a}{\partial x_{l}}(x(t), \xi) e^{2 \pi i(x(t)-y) \cdot \xi} \\
& +2 \pi i \xi_{l} a(x(t), \xi) e^{2 \pi i(x(t)-y) \cdot \xi} d t
\end{aligned}
$$

where $x(t)=z+t(x-z)$.

Using this last expression and the facts:

(a) $\partial a / \partial x_{l}$ is a symbol of order 0 ;

(b) $\xi_{l} a(x, \xi)$ is a symbol of order 1 ;

(c) $\left|x_{l}-z_{l}\right| \leqslant d$ since both $x$ and $z$ are in $Q$; and

(d) if $2^{k} d \leqslant\left|y-x^{\prime}\right| \leqslant 2^{k+1} d$, then $2^{k-1} d \leqslant|x(t)-y|<2^{k+2} d$ since $x(t) \in Q$, we can invoke Lemma 2.9 with $m=0$ or 1 , and $t=n+\frac{1}{2}$ to see that (2.11) is dominated by

$$
\begin{aligned}
& C \sup _{x \in Q} \sum_{k=1}^{\infty} \frac{1}{|Q|} \int_{Q} \int_{2^{k} d<\left|y-x^{\prime}\right|<2^{k+1} d} \frac{|u(y)|}{\left|y-x^{\prime}\right|^{n+1 / 2}} \\
& \quad \cdot \sum_{l=1}^{n}\left|x_{l}-z_{l}\right| \int_{0}^{1}|x(t)-y|^{n+1 / 2} \\
& \cdot\left|\int_{R^{n}} \phi\left(2^{-j} \xi\right)\left[\frac{\partial a}{\partial x_{l}}(x(t), \xi) e^{2 \pi i(x(t)-y) \cdot \xi}+2 \pi i \xi_{l} a(x(t), \xi) e^{2 \pi i(x(t)-y) \cdot \xi}\right] d \xi\right| d t d y d z \\
& \leqslant C \sum_{k=1}^{\infty} 2^{n k} \frac{1}{\left|Q_{k}\right|} \int_{Q_{k}}|u(y)| d y d^{n}\left(2^{k} d\right)^{-n-1 / 2} d\left(2^{-j / 2}+2^{j / 2}\right) \\
& \leqslant C M u\left(x^{0}\right) d^{1 / 2} 2^{j / 2} \sum_{k=1}^{\infty} 2^{-k / 2} \\
& \leqslant C d^{1 / 2} 2^{j / 2} M u\left(x^{0}\right) .
\end{aligned}
$$

Putting the two cases together, we have shown that if $Q$ is any cube containing $x^{0}$, then

$$
\begin{aligned}
\frac{1}{|Q|} \int_{Q}\left|\sum_{j=0}^{\infty} A_{j} u(x)-\left(\sum_{j=0}^{\infty} A_{j} u\right)_{Q}\right| d x \leqslant \sum_{j=0}^{\infty} \frac{1}{|Q|} \int_{Q}\left|A_{j} u(x)-\left(A_{j} u\right)_{Q}\right| d x \\
\leqslant C\left(\sum_{2^{\prime} d>1} d^{-1} 2^{-j}+\sum_{2^{j} d<1} d^{1 / 2} 2^{j / 2}\right) M u\left(x^{0}\right) .
\end{aligned}
$$

Since the quantity in parentheses above is finite and independent of $d$, we find, after taking the supremum over all cubes $Q$ containing $x^{0}$, that

$$
\left(\sum_{j=0}^{\infty} A_{j} u\right)^{\#}\left(x^{0}\right) \leqslant c M u\left(x^{0}\right) \leqslant c M_{r} u\left(x^{0}\right) \text {. }
$$


Going back to our original notation, and summarizing, we have shown that if $Q$ is any cube containing $x^{0}$, then

$$
\begin{aligned}
\frac{1}{|Q|} \int_{Q}\left|A u(x)-(A u)_{Q}\right| d x \leqslant & (A(\tau u))^{\#}\left(x^{0}\right)+(B((1-\tau) u))^{\#}\left(x^{0}\right) \\
& +\left(\sum_{j=0}^{\infty} A_{j}((1-\tau) u)\right)^{\#}\left(x^{0}\right) \\
\leqslant & \leqslant M_{r} u\left(x^{0}\right)+c M_{r}((1-\tau) u)\left(x^{0}\right) \\
& \leqslant c M_{r} u\left(x^{0}\right),
\end{aligned}
$$

the constant $c$ being independent of $Q, u, x^{0}$, and the $\xi$-support of $a(x, \xi)$.

We have been working under the assumption that $a(x, \xi)$, the symbol of $A$, has compact $\xi$-support. Suppose now that this is no longer so. Let $b_{j}(x, \xi)$ be $a(x, \xi)$ multiplied by a smooth cutoff function which is 1 when $|\xi| \leqslant 2^{j}$ and 0 when $|\xi| \geqslant 2^{j+1}$. Let $B_{j}$ be the pseudodifferential operator whose symbol is $b_{j}(x, \xi)$. Since $b_{j}(x, \xi) \rightarrow a(x, \xi)$ as $j \rightarrow \infty$, the dominated convergence theorem implies that $B_{j} u(x) \rightarrow A u(x)$ for all $x$. Another application of the dominated convergence theorem shows that for each cube $Q$,

$$
\frac{1}{|Q|} \int_{Q}\left|B_{j} u(x)-\left(B_{j} u\right)_{Q}\right| d x \rightarrow \frac{1}{|Q|} \int_{Q}\left|A u(x)-(A u)_{Q}\right| d x .
$$

Applying our previous result to the operators $B_{j}$, and taking the limit as $j \rightarrow \infty$, we see that

$$
\frac{1}{|Q|} \int_{Q}\left|A u(x)-(A u)_{Q}\right| d x \leqslant c M_{r} u\left(x^{0}\right) .
$$

When we take the supremum of the left side over all cubes containing $x^{0}$, we finally obtain the inequality

$$
(A u)^{\#}\left(x^{0}\right) \leqslant c M_{r} u\left(x^{0}\right) .
$$

We are now ready to prove a basic result about pseudodifferential operators.

THEOREM 2.12. If $1<p<\infty$ and $w \in A_{p}\left(R^{n}\right)$, then any pseudodifferential operator of order 0 has a bounded extension to all of $L^{p}\left(R^{n}, w d x\right)$.

Proof. Let $A$ be a pseudodifferential operator of order 0 . The proof that $A$ is bounded depends on the following train of inequalities: if $u \in \mathcal{S}$ then

$$
\begin{aligned}
\|A u\|_{p} & \leqslant\left\|(A u)^{*}\right\|_{p} \leqslant C\left\|(A u)^{\#}\right\|_{p} \\
& \leqslant C\left\|M_{r} u\right\|_{p} \quad \text { if } 1<r<\infty \\
& \leqslant C\|u\|_{p} \quad \text { if } 1<r<p .
\end{aligned}
$$

The first inequality is easy, since $|A u(x)| \leqslant(A u)^{*}(x)$ for every $x$. Since $A u \in \mathcal{S}$, $A u \in L^{p}\left(R^{n}, w d x\right) \cap L^{1}\left(R^{n}, d x\right)$; so we can apply Lemma 2.7 to prove the 
second inequality. The third inequality is Theorem 2.8 , while the last inequality is proved like this:

$$
\begin{aligned}
\left\|M_{r} u\right\|_{p} & =\left\|\left[M\left(|u|^{r}\right)\right]^{1 / r}\right\|_{p}=\left(\int\left[M\left(|u|^{r}\right)\right]^{p / r} w d x\right)^{1 / p} \\
& \leqslant C\left(\int|u|^{p} w d x\right)^{1 / p} \text { since } p / r>1 \\
& =C\|u\|_{p} .
\end{aligned}
$$

Since $\mathcal{S}$ is dense in $L^{p}\left(R^{n}, w d x\right)$, we can now extend $A$ to a bounded operator on $L^{p}\left(R^{n}, w d x\right)$.

3. Weighted Sobolev spaces in $R^{n}$. In this section we shall introduce the weighted Sobolev spaces $L_{s}^{p}\left(R^{n}, w d x\right)$. It will transpire that many of the properties of the traditional unweighted spaces are still true in the weighted case; in particular, we can identify the space $L_{k}^{p}\left(R^{n}, w d x\right)$ ( $k$ a positive integer) with the space of functions in $L^{p}\left(R^{n}, w d x\right)$ whose distributional derivatives of all orders $\leqslant k$ lie in $L^{p}\left(R^{n}, w d x\right)$, and we can prove a version of Sobolev's theorem.

If $s$ is any real number, we write $J^{s}$ for the pseudodifferential operator of order $-s$ whose symbol is $\left(1+4 \pi^{2}|\xi|^{2}\right)^{-s / 2}$. Clearly, $J^{s}$ can be defined as a map of tempered distributions to tempered distributions; we also point out that if $w \in$ $A_{p}\left(R^{n}\right)$, then functions in $L^{p}\left(R^{n}, w d x\right)$ are tempered distributions. We define $L_{s}^{p}\left(R^{n}, w d x\right)$, the Sobolev space of order $s$, as the image of $L^{p}\left(R^{n}, w d x\right)$ under the map $J^{s}$; i.e., $L_{s}^{p}\left(R^{n}, w d x\right)=J^{s}\left(L^{p}\left(R^{n}, w d x\right)\right)$. If $f \in L_{s}^{p}\left(R^{n}, w d x\right)$, then $f=J^{s} g$ for some $g \in L^{p}\left(R^{n}, w d x\right)$. We write the $L_{s}^{p}$-norm of $f$ as $\|f\|_{p, s}$, and define it to be the $L^{p}$-norm of its preimage $g$. So $\|f\|_{p, s}=\|g\|_{p}$ whenever $f=J^{s} g$.

The following facts about the $L_{s}^{p}$ are easy consequences of the definitions.

(a) Since $J^{s}$ is an invertible elliptic pseudodifferential operator, the definition of the norm on $L_{s}^{p}\left(R^{n}, w d x\right)$ is unambiguous; i.e., if $J^{s} g_{1}=J^{s} g_{2}$ then $g_{1}=g_{2}$.

(b) If $s \geqslant 0$, then $L_{s}^{p}\left(R^{n}, w d x\right)$ is a subspace of $L^{p}\left(R^{n}, w d x\right)$, since $J^{s}$ is a pseudodifferential operator of order 0 .

(c) For all real $s$ and $t, J^{s} J^{t}=J^{s+t}$.

(d) For all real $s, J^{s}$ is an isomorphism of $\mathcal{S}$ to $\delta$ and of $\mathcal{S}^{\prime}$ to $\mathcal{S}^{\prime}$; furthermore, $\mathcal{S}$ is dense in $L_{s}^{p}\left(R^{n}, w d x\right)$.

(e) For all real $s$ and $t, J^{t}$ is a norm-preserving isomorphism of $L_{s}^{p}\left(R^{n}, w d x\right)$ to $L_{s+t}^{p}\left(R^{n}, w d x\right)$.

(f) The spaces $L_{s}^{p}\left(R^{n}, w d x\right)$ are Banach spaces.

(g) If $s \geqslant t$ then $L_{s}^{p}\left(R^{n}, w d x\right) \subseteq L_{t}^{p}\left(R^{n}, w d x\right)$, and $\|f\|_{p, t} \leqslant C_{s, t}\|f\|_{p, s}$.

That pseudodifferential operators behave correctly on Sobolev spaces is the content of the next two theorems.

THEOREM 3.1. Suppose $A$ is a pseudodifferential operator of order $m$. Then $A$ is $a$ bounded map from $L_{s}^{p}\left(R^{n}, w d x\right)$ to $L_{s-m}^{p}\left(R^{n}, w d x\right)$.

Proof. We can write $A=J^{s-m}\left(J^{-s+m} A J^{s}\right) J^{-s} . J^{-s}$ maps $L_{s}^{p}$ to $L^{p} ; J^{-s+m} A J^{s}$ is a pseudodifferential operator of order 0 , and therefore maps $L^{p}$ to $L^{p}$; finally, $J^{s-m}$ maps $L^{p}$ to $L_{s-m}^{p}$. 
THEOREM 3.2. Let $0 \leqslant m \leqslant s$ and suppose that $A$ is an elliptic pseudodifferential operator of order $m$. Then there is a constant $c_{s}>0$ such that

$$
\|f\|_{p, s} \leqslant c_{s}\left(\|A f\|_{p, s-m}+\|f\|_{p, 0}\right), \quad f \in L_{s}^{p} .
$$

Proof. Since $A$ is elliptic, we can find an elliptic operator $B$, of order $-m$, and an operator $R$, of order $-\infty$, such that $I$, the identity operator, can be written $I=B A+R$. Theorem 3.1 then shows that

$$
\begin{aligned}
\|f\|_{p, s} & =\|(B A+R) f\|_{p, s} \leqslant\|B A f\|_{p, s}+\|R f\|_{p, s} \\
& \leqslant c_{s}\left(\|A f\|_{p, s-m}+\|f\|_{p, 0}\right) . \quad \square
\end{aligned}
$$

The expected identification theorem is also true.

THEOREM 3.3. Suppose $k$ is a positive integer and $1<p<\infty$. The space $L_{k}^{p}\left(R^{n}, w d x\right)$ is identical to the subspace of functions in $L^{p}\left(R^{n}, w d x\right)$ whose distributional derivatives of all orders $\leqslant k$ lie in $L^{p}\left(R^{n}, w d x\right)$. Furthermore, the norms $\|f\|_{p, k}$ and $\Sigma_{|\alpha| \leqslant k}\left\|(\partial / \partial x)^{\alpha} f\right\|_{p}$ are equivalent.

Proof. We can use the same proof as Theorem 7 of [1], if we keep in mind that $(\partial / \partial x)^{\alpha} J^{k}$ is a pseudodifferential operator of order 0 , and hence bounded on $L^{p}\left(R^{n}, w d x\right)$ whenever $|\alpha| \leqslant k$.

As in the unweighted case, the weighted Sobolev spaces can be used to compare the size of the distributional derivatives of a function and its degree of smoothness. The following is a weak form of Sobolev's theorem.

THEOREM 3.4. Suppose that $w \in A_{q}\left(R^{n}\right)$ for some $q$ satisfying $1<q<$ $p(n-1) / n$. If $k>n q / p$, then every function in $L_{k}^{p}\left(R^{n}, w d x\right)$ can be modified on a set of measure 0 so that the resulting function is continuous.

Proof. Fix $f \in L_{k}^{p}\left(R^{n}, w d x\right)$, and suppose that $f=J^{k} g$, with $g \in L^{p}\left(R^{n}, w d x\right)$. Let $\left\{g_{n}\right\}$ be a sequence in $\mathcal{S}$ such that $g_{n} \rightarrow g$ in $L^{p}\left(R^{n}, w d x\right)$ (Lemma 2.1), and let $f_{n}=J^{k} g_{n}$. If $|\alpha| \leqslant k$, then $(\partial / \partial x)^{\alpha} J^{k}$ is a pseudodifferential operator of order 0 ; consequently,

$$
\left(\frac{\partial}{\partial x}\right)^{\alpha} f_{n}=\left(\frac{\partial}{\partial x}\right)^{\alpha} J^{k} g_{n} \rightarrow\left(\frac{\partial}{\partial x}\right)^{\alpha} J^{k} g=\left(\frac{\partial}{\partial x}\right)^{\alpha} f
$$

the limit being taken in $L^{p}\left(R^{n}, w d x\right)$. So $f_{n} \rightarrow f$ in $L_{k}^{p}\left(R^{n}, w d x\right)$ by Theorem 3.3.

Since $n q / p<n-1$, we can assume, by decreasing $k$ if necessary, that $k$ is an integer and that $n q / p<k \leqslant n-1$. Let $K$ be any compact set. If $\phi$ is a function in $C_{0}^{\infty}\left(R^{n}\right)$ which is identically 1 on $K$, it is clearly enough to prove the theorem for $\phi f$. Since the sequence $\left\{f_{n}\right\}$ approximates $f$ in $L_{k}^{p}\left(R^{n}, w d x\right)$, the sequence $\left\{\phi f_{n}\right\}$ approximates $\phi f$.

Now choose $R$ large enough that if $K_{1}$ is the support of $\phi$, then the set $K_{1}-K_{1}$ is contained in the ball of radius $R$ about the origin. If $x \in K$, we use identity (18) on page 125 of [9] repeatedly to write

$$
\begin{aligned}
\left|f_{j}(x)-f_{l}(x)\right| & =\left|\phi(x)\left(f_{j}(x)-f_{l}(x)\right)\right| \\
& \leqslant C \sum_{|\alpha|=k}\left|\frac{\partial^{\alpha}\left[\phi\left(f_{j}-f_{l}\right)\right]}{\partial x^{\alpha}}\right| * g * g * \cdots * g(x),
\end{aligned}
$$


where there are $k$ repetitions of $g(x)=|x|^{-n+1}$. By Lemma 2 on page 117 of [9],

$$
g * g * \cdots * g(x)=C|x|^{-n+k} \quad(k \text { repetitions of } g) \text {. }
$$

Hence,

$$
\begin{aligned}
\mid f_{j}(x)- & \left.f_{l}(x)\left|\leqslant C \sum_{|\alpha|=k} \int_{|y|<R}\right| \frac{\partial^{\alpha}\left[\phi\left(f_{j}-f_{l}\right)\right]}{\partial x^{\alpha}}(x-y)|| y\right|^{-n+k} d y \\
& \leqslant C \sum_{|\alpha|=k}\left\|\frac{\partial^{\alpha}\left[\phi\left(f_{j}-f_{l}\right)\right]}{\partial x^{\alpha}}\right\|_{p}\left(\int_{|y|<R} w(x-y)^{-1 /(p-1)}|y|^{(-n+k) p^{\prime}} d y\right)^{1 / p^{\prime}} .
\end{aligned}
$$

If we can show that the second factor on the right side of the last inequality is bounded independently of $x \in K$, it will follow that the $\left\{f_{n}\right\}$ converge uniformly on $K$, and therefore that $f$ is continuous on $K$. Therefore, let $q$ be as in the statement of the theorem, and set $r=(p-1) /(q-1)$. Since $r>1$, we can apply Hölder's inequality to the integral in question to find that

$$
\begin{aligned}
\int_{|y|<R} w(x-y)^{-1 /(p-1)}|y|^{(-n+k) p^{\prime}} d y & \\
& \quad<\left(\int_{|y|<3 R} w(y)^{-1 /(q-1)} d y\right)^{1 / r}\left(\int_{|y|<R}|y|^{(-n+k) p /(p-q)} d y\right)^{1 / r^{\prime}} .
\end{aligned}
$$

Since $w \in A_{q}\left(R^{n}\right)$, the first integral on the right side is finite. The second integral is finite since $(-n+k) p /(p-q)>-n$.

EXAMPLE 3.5. Suppose $q>1$, and let $-n<t<n(q-1)$. It is well known that $|x|^{t}$ is in $A_{q}\left(R^{n}\right)$, and hence in $A_{p}\left(R^{n}\right)$, for all $p>q$. Now choose $p>n q /(n-1)$. By Theorem 3.4, every function in $L_{n-1}^{p}\left(R^{n},|x|^{t} d x\right)$ is actually continuous.

4. Weighted Sobolev spaces on manifolds. We can now transfer the results of the preceding sections to a compact manifold without boundary. This requires that we localize the previous results to bounded open subsets of $R^{n}$. If $U$ and $V$ are two open subsets of $R^{n}$, the notation $U \subset \subset V$ will mean that the closure of $U$ is compact and contained inside $V$.

Suppose $U$ is a bounded open subset of $R^{n}$. The function $w$ is said to belong to $A_{p}(U)$ if

(i) $w$ is nonnegative and integrable over every compact subset of $U$;

(ii) for every open set $U^{\prime} \subset \subset U$, there is a constant $C$, which may depend on $U^{\prime}$, such that

$$
\left(\frac{1}{|Q|} \int_{Q} w d x\right)\left(\frac{1}{|Q|} \int_{Q} w^{-1 /(p-1)} d x\right)^{p-1} \leqslant C
$$

whenever $Q$ is a cube contained in $U^{\prime}$. The constant $C$ will be called the $A_{p}$ constant for $U^{\prime}$.

The next lemma shows that the $A_{p}$ condition is invariant under coordinate changes.

Lemma 4.1. Suppose $U$ and $V$ are bounded open sets in $R^{n}$, and $\psi: U \rightarrow V$ is a diffeomorphism. If $w \in A_{p}(V)$ then $w \circ \psi \in A_{p}(U)$. 
Proof. Suppose $U^{\prime} \subset \subset U$; then $V^{\prime}=\psi\left(U^{\prime}\right) \subset \subset V$. Using $x$ to denote the coordinates in $U$, and $y$ to denote the coordinates in $V$, we have $x=\psi^{-1}(y)$ and $d x=\left|J \psi^{-1}(y)\right| d y$, where $J \psi^{-1}$ is the Jacobian determinant of $\psi^{-1}$. Let $V^{\prime \prime}$ be an open set such that $V^{\prime} \subset \subset V^{\prime \prime} \subset \subset V$. The following list of constants will be used in the remainder of the proof:

$b=\sup \left\{\left|J \psi^{-1}(y)\right|: y \in V^{\prime}\right\}$,

$\delta=$ the distance from $V^{\prime}$ to the complement of $V^{\prime \prime}$,

$d=$ the $A_{p}$ constant for $V^{\prime \prime}$,

$k=\sup \left\{|D \psi(x)|: x \in U^{\prime}\right\}$, where $D \psi$ is the differential of $\psi$.

Now suppose $S$ is a cube in $U^{\prime}$ with center $x^{0}$ and side length $2 r$, with $r<\delta / k n$. If $x$ is any point in $S$, then

$$
\begin{aligned}
\left|\psi(x)-\psi\left(x^{0}\right)\right| & \leqslant k\left|x-x^{0}\right| \text { for the Mean Value Theorem } \\
& \leqslant k r \sqrt{n} \leqslant \delta / \sqrt{n} .
\end{aligned}
$$

Hence $\psi(S)$ is contained inside any cube with center $\psi\left(x^{0}\right)$ and side length $2 k \sqrt{n} r$, and any such cube lies entirely inside $V^{\prime \prime}$. Let $Q$ be such a cube in $V^{\prime \prime}$. Then

$$
\begin{aligned}
\left(\frac{1}{|S|} \int_{S} w \circ \psi(\right. & x) d x)\left(\frac{1}{|S|} \int_{S} w \circ \psi(x)^{-1 /(p-1)} d x\right)^{p-1} \\
& \leqslant\left(\frac{b(2 k \sqrt{n})^{n}}{|Q|} \int_{Q} w(y) d y\right)\left(\frac{b(2 k \sqrt{n})^{n}}{|Q|} \int_{Q} w(y)^{-1 /(p-1)} d y\right)^{p-1} \\
& \leqslant b^{p}(2 k \sqrt{n})^{n p} d .
\end{aligned}
$$

The $A_{p}$ condition is therefore proved for small cubes in $U^{\prime}$ with side length $<2 \delta / k n$.

Now suppose that $S$ is a cube in $U^{\prime}$ with side length $2 r \geqslant 2 \delta / k n$. Then

$$
\begin{aligned}
\left(\frac{1}{|S|} \int_{S} w \circ \psi(x)\right. & d x)\left(\frac{1}{|S|} \int_{S} w \circ \psi(x)^{-1 /(p-1)} d x\right)^{p-1} \\
& \leqslant\left(\left(\frac{k n}{2 \delta}\right)^{n} \int_{U^{\prime}} w \circ \psi(x) d x\right)\left(\left(\frac{k n}{2 \delta}\right)^{n} \int_{U^{\prime}} w \circ \psi(x)^{-1 /(p-1)} d x\right)^{p-1} \\
& \left(\frac{k n}{2 \delta}\right)^{n p} b^{p}\left(\int_{V^{\prime}} w(y) d y\right)\left(\int_{V^{\prime}} w(y)^{-1 /(p-1)} d y\right)^{p-1} \\
\leqslant & \text { since } w \text { and } w^{-1 /(p-1)} \text { are integrable on } V^{\prime} .
\end{aligned}
$$

The result that $w \circ \psi \in A_{p}(U)$ now follows.

We now formulate the $A_{p}$ condition for a $C^{\infty}$ compact manifold without boundary. A word about notation: we say that $(\Omega, \phi)$ is a coordinate chart when $\Omega$ is a coordinate neighborhood on the manifold, and $\phi$ is a $C^{\infty}$ coordinate map from $\Omega$ to some open subset of $R^{n}$.

Let $M$ be a compact $C^{\infty}$ manifold without boundary, and let $\left\{\left(\Omega_{i}, \phi_{i}\right)\right\}_{i=1}^{k}$ be a fixed finite atlas for $M$. If $w$ is a nonnegative function on $M$, then $w \in A_{p}(M)$ if $w \circ \phi_{i}^{-1} \in A_{p}\left(\phi_{i}\left(\Omega_{i}\right)\right)$ for $i=1,2, \ldots, k$. 
THEOREM 4.2. The definition of $A_{p}(M)$ is independent of the particular atlas $\left\{\left(\Omega_{i}, \phi_{i}\right)\right\}_{i=1}^{k}$. That is, $w \in A_{p}(M)$ if and only if $w \circ \phi^{-1} \in A_{p}(\phi(\Omega))$ for any coordinate chart $(\Omega, \phi)$.

Proof. One implication is obvious; so we shall assume that $w \in A_{p}(M)$ and that $(\Omega, \phi)$ is a randomly chosen coordinate chart. Suppose $U \subset \subset \phi(\Omega)$, and let $Q$ be a cube in $U$. Since $\operatorname{cl}(U)$, the closure of $U$, is contained in $U_{i} \phi\left(\Omega_{i} \cap \Omega\right)$, we can pick open sets $W_{i}$ in $R^{n}$ such that $W_{i} \subset \subset \phi\left(\Omega_{i} \cap \Omega\right)$ and $\operatorname{cl}(U) \subset \cup_{i} W_{i}$. When $\left\{W_{i}\right\}_{i=1}^{k}$ is regarded as a covering of $\operatorname{cl}(U)$, it has a Lebesgue number, $l$. If $Q$ has diameter $<l$, then it lies entirely inside $W_{i}$ for some $i$. Since $\phi_{i} \circ \phi^{-1}$ is a diffeomorphism between $\phi\left(\Omega \cap \Omega_{i}\right)$ and $\phi_{i}\left(\Omega \cap \Omega_{i}\right)$, and since $W_{i} \subset \varnothing\left(\Omega \cap \Omega_{i}\right)$, the last lemma shows that the $A_{p}$ condition holds for all cubes with diameter $<l$.

On the other hand, if $Q$ is a cube inside $U$ with diameter $>l$ then

$$
\begin{aligned}
\left(\frac{1}{|Q|} \int_{Q} w \circ \phi^{-1} d x\right) & \left(\frac{1}{|Q|} \int_{Q}\left[w \circ \phi^{-1}\right]^{-1 /(p-1)} d x\right)^{p-1} \\
\leqslant & \frac{1}{l^{n p}}\left(\int_{U} w \circ \phi^{-1} d x\right)\left(\int_{U}\left[w \circ \phi^{-1}\right]^{-1 /(p-1)} d x\right)^{p-1} \\
\leqslant & \frac{C}{l^{n p}}\left(\sum_{i=1}^{k} \int_{\phi_{i} \circ \phi^{-1}\left(W_{i}\right)} w \circ \phi_{i}^{-1} d y\right) \\
& \cdot\left(\sum_{i=1}^{k} \int_{\phi_{i} \circ \phi^{-1}\left(W_{i}\right)}\left[w \circ \phi_{i}^{-1}\right]^{-1 /(p-1)} d y\right)^{p-1} \\
\leqslant & C .
\end{aligned}
$$

Having fixed the particular atlas $\left\{\left(\Omega_{i}, \phi_{i}\right)\right\}$, we now choose a nonnegative $C^{\infty}$ partition of unity, $\left\{\tau_{i}\right\}$, subordinate to this atlas. If $w \in A_{p}(M)$ is restricted to $\Omega_{i}$, we can regard it as a function in $A_{p}\left(\phi_{i}\left(\Omega_{i}\right)\right)$. Similarly, if $f$ is a function defined on $M$, we will consider $\tau_{i} f$ as a function with compact support defined in $R^{n}$. We say that $f \in L^{p}(M, w d x)$ if $\tau_{i} f \in L^{p}\left(\phi_{i}\left(\Omega_{i}\right), w d x\right)$ for each $i$. The norm on $L^{p}(M, w d x)$ is given by

$$
\|f\|_{p}=\left(\sum_{i=1}^{k}\left\|\tau_{i}^{1 / p}\right\|_{p}^{p}\right)^{1 / p},
$$

where the norms on the right side are given by

$$
\left\|\tau_{i}^{1 / p} f\right\|_{p}=\left(\int_{\phi_{i}\left(\Omega_{i}\right)} \tau_{i}|f|^{p} w d x\right)^{1 / p}
$$

A standard argument shows that the norms given by different atlases and different partitions of unity are all equivalent and define the same topology on $L^{p}(M, w d x)$.

We recall that a pseudodifferential operator can be defined on the manifold $M$ by prescribing the action of the operator on functions supported in a coordinate patch. The operator $A$ is said to be of order $m$ if in each coordinate patch $\Omega$ we can write $A u(x)=\int \hat{u}(\xi) a(x, \xi) e^{2 \pi i x \cdot \xi} d \xi$, with $a(x, \xi)$ a symbol of order $m$, whenever 
$x \in \Omega$ and $u$ is a function supported in $\Omega$. By using a partition of unity subordinate to the covering by coordinate charts, we can extend the definition of $A$ to all functions in $C^{\infty}(M)$. (See, e.g., [7].)

The next theorem allows us to localize the estimates of Theorem 2.12.

THEOREM 4.3. Suppose $U$ is a bounded open subset of $R^{n}$, and let $w \in A_{p}(U)$. If $V \subset \subset U$ and if $A$ is a pseudodifferential operator of order 0 , then $A: L^{p}(V, w d x) \rightarrow$ $L^{p}(V, w d x)$ boundedly.

Proof. Since $V \subset \subset U$, we can cover $V$ with a finite number of cubes $\left\{Q_{i}\right\}_{i=1}^{k}$ such that $Q_{i} \subset \subset U$. By introducing a partition of unity subordinate to these cubes, we need only show that $A: L^{p}\left(Q_{i}, w d x\right) \rightarrow L^{p}\left(Q_{i}, w d x\right)$ boundedly for $i=$ $1,2, \ldots, k$.

So we choose one of the cubes $Q$ and, by translating it in the directions of its edges, we decompose $R^{n}$ into a mesh of cubes the same size as $Q$, whose interiors are disjoint, and whose sides are parallel to those of $Q$.

The next step is to extend the function $w$ from $Q$ to the rest of $R^{n}$. We do this by reflecting the values of $w$ through the sides of $Q$ into its adjacent cubes, continuing in this way so that the values of $w$ in cubes sharing a common face match up along that face. The resulting function $w^{\prime}$ lies in $A_{p}\left(R^{n}\right)$, and has an $A_{p}$ constant no more than $3^{n}$ times the $A_{p}(V)$ constant for $w$.

The rest is easy, since if $u$ is supported in $Q$, then

$$
\|A u\|_{p, Q} \leqslant\|A u\|_{p, R^{n}} \leqslant C\|u\|_{p, R^{n}}=C\|u\|_{p, Q}
$$

where the first and last norms are in $L^{p}(Q, w d x)$ and the middle two are in $L^{p}\left(R^{n}, w^{\prime} d x\right)$.

Corollary 4.4. If $A$ is a pseudodifferential operator of order 0 , and $w \in A_{p}(M)$, then $A: L^{p}(M, w d x) \rightarrow L^{p}(M, w d x)$ boundedly.

PRoOF. Use a partition of unity subordinate to a coordinate covering of $\boldsymbol{M}$.

Now we can define $L_{s}^{p}(M, w d x)$, the Sobolev potential space of order $s$ on the manifold $M$. Let $E_{s}$ be an invertible elliptic pseudodifferential operator of order $s$ defined on $C^{\infty}(M) . L_{s}^{p}(M, w d x)$ is the set of all distributions $f$ defined on $C^{\infty}(M)$ such that $E_{s} f \in L^{p}(M, w d x)$. We define a norm on this space by $\|f\|_{p, s}=\left\|E_{s} f\right\|_{p}$.

At first glance, it seems as if $L_{s}^{p}(M, w d x)$ depends on the choice of $E_{s}$, but this is not so. For suppose that $E$ is another invertible elliptic pseudodifferential operator of order $s$. Then

$$
\|f\|_{p, s}=\left\|E_{s}\right\|_{p}=\left\|E_{s} E^{-1} E f\right\|_{p} \leqslant C\|E f\|_{p},
$$

since $E_{s} E^{-1}$ is a pseudodifferential operator of order 0 , and hence bounded on $L^{p}(M, w d x)$. The norms defined by different $E$ 's are therefore all equivalent.

By introducing a partition of unity, covering coordinate patches in $R^{n}$ with cubes, and extending $w$ from the cubes to all of $R^{n}$ as in the proof of the last theorem, we can transfer all the results stated in $\$ 3$ to $L_{s}^{p}(M, w d x)$. We list some of these results in our last theorem. 
THEOREM 4.5. Let $M$ be a compact $C^{\infty}$ manifold without boundary, and let $w \in A_{p}(M)$.

(a) The spaces $L_{s}^{p}(M, w d x)$ are Banach spaces.

(b) If $s \geqslant t$ then $L_{s}^{p} \subseteq L_{t}^{p}$ and $\|f\|_{p, t} \leqslant C\|f\|_{p, s^{*}}$.

(c) Suppose $A$ is a pseudodifferential operator of order $m \leqslant s$. Then $A: L_{s}^{p} \rightarrow L_{s-m}^{p}$ boundedly.

(d) If $A$ is an elliptic pseudodifferential operator of order $m$, and $0 \leqslant m \leqslant s$, then there is a constant $C_{s}>0$ such that

$$
\|u\|_{p, s} \leqslant C_{s}\left(\|A u\|_{p, s-m}+\|u\|_{p}\right), \quad u \in L_{s}^{p} .
$$

(e) Suppose $k$ is a positive integer. The space $L_{k}^{p}(M, w d x)$ coincides with the subspace of functions in $L^{p}(M, w d x)$ having distributional derivatives of all orders $\leqslant k$ in $L^{p}(M, w d x)$ in any coordinate system.

(f) Suppose that $w \in A_{q}(M)$ for some $q$ satisfying $1<q<p(n-1) / n$. If $s>$ $n q / p$, then every function in $L_{s}^{p}(M, w d x)$ is continuous.

\section{REFERENCES}

1. A. P. Caldéron, Lebesgue spaces of differentiable functions and distributions, Proc. Sympos. Pure Math., Vol. 4, Amer. Math. Soc., Providence, R. I., 1961.

2. R. Coifman and C. Fefferman, Weighted norm inequalities for maximal functions and singular integrals, Studia Math. 51 (1974), 241-250.

3. C. Fefferman and E. M. Stein, $H^{p}$ spaces of several variables, Acta Math. 129 (1972), 137-193.

4. R. A. Hunt, B. Muckenhoupt and R. L. Wheeden, Weighted norm inequalities for the conjugate function and Hilbert transform, Trans. Amer. Math. Soc. 176 (1973), 227-251.

5. R. Illner, $A$ class of $L^{p}$-bounded pseudo-differential operators, Proc. Amer. Math. Soc. 51 (1975), 347-355.

6. B. Muckenhoupt, Weighted norm inequalities for the Hardy-Littlewood maximal function, Trans. Amer. Math. Soc. 165 (1972), 207-226.

7. L. Nirenberg, Pseudo-differential operators, Proc. Sympos. Pure Math., Vol. 16, Amer. Math. Soc., Providence, R. I., 1970.

8. W. Rudin, Real and complex analysis, McGraw-Hill, New York, 1966.

9. E. M. Stein, Singular integrals and differentiability properties of functions, Princeton Univ. Press, Princeton, N. J., 1970.

Department of Mathematics, Gustavus Adolphus College, St. Peter, Minnesota 56082 